\title{
Nanotheranostics-An Emerging Technique in Nanomedicine
}

\author{
Nida Tabassum khan* \\ Department of Biotechnology, Balochistan University of Information Technology, Pakistan \\ *Corresponding author: Nida tabassum khan, Department of Biotechnology, Balochistan University of Information Technology, \\ Pakistan. \\ To Cite This Article: Nida Tabassum khan. Nanotheranostics-An Emerging Technique in Nanomedicine. Am J Biomed Sci \& Res. 2021 - 14(1). \\ AJBSR.MS.ID.001945. DOI: 10.34297/AJBSR.2021.14.001945.
}

Received: 眥 August 23, 2021; Published: 眥 August 27, 2021

\begin{abstract}
Nanotheranostics is a novel yet developing field which promises advanced methods for clinical diagnostics and treatment. Certain biological molecules present inside human body and their varying concentrations are used as detectable signals for nanoparticles or nanoconjugates to identify cancerous or tumors cells and also targeting these effected cells for the treatment of disease. At present, available treatments are expensive, time consuming with potential side effects. Therefore, application of nanotheranostics will surely help to minimize such issues and promises better therapeutics in nanomedicine
\end{abstract}

Keywords: Diagnostic; Surface Plasmon Resonance; Nanosensors; Radiotherapy; Photosensitizer

\section{Introduction}

The word theranostics means when the efforts of diagnostic imaging and therapy are combined together [1]. It can be used in clinical diagnostics, disease determination of early progression of disease, selection of therapy, prognosis and follow-ups [2,3]. It can be used in personalized particularly for the treatment of cancer [4]. The main role in which combines diagnostics and therapeutics to design personalized medicine for various devastating diseases like cancer and tumor's $[5,6]$. Nanotheranostics can be used to identify biomarkers for cancer diagnosis and the same molecules can be targeted for therapeutic purpose as well [7]. Use of nanosized particles in diagnostics helps in designing specific nanosensors for disease identification and treatment in nanomedicine [8]. These nanosensors are capable of detecting specific biomarkers in clinical samples for targeted drug delivery [9]. At present, available treatments are expensive, time consuming with potential side effects. Therefore, application of nanotheranostics will surely help to minimize such issues [10]. However, this technology is yet to be fully developed because diseases are diverse with different microenvironment, so it requires different nanomaterials to meet the growing need for better treatment [11]. There are some unique properties of nanoparticles that make them great for clinical purposes such as their small size and high surface area to volume ratio enables targeted drug delivery in vivo [12].In addition, intrinsic imaging properties of specific nanoparticles can be utilized for nanotheranostics applications [13].These properties are highly helpful in screening and diagnosis of different molecules associated with molecularly complex diseases like cancer, autoimmune disorders and neurodegenerative diseases [14,15].

\section{Types of Nanomaterials Used in Nanotheranostics}

a) Gold Nanoparticles: Gold nanoparticles of various structural conformation such as nanocages, nano shells or nanorods could be employed in nanotheranostics [16]. These differential structural conformation of gold nanoparticles contributes for distinct localized surface plasmon resonance (LSPR) property [17], a characteristic which plays an important role in nanotheranostics particularly in killing tumorous/cancerous cells by using laser energy set to a particular LSPR which will excite the nanoparticles resulting in instantaneous release of thermal energy that will destroy the targeted cancerous cells [18]. On the other hand, photothermal therapy can also be used at targeted 
tumorous regions that cannot be operated with surgeries by employing laser beam set at infrared regions to penetrate body deep tissues [19]. Besides being used in nanotherapeutics, nanoparticles are also employed in imaging diagnostics such as gold nanoparticles fluorescent-quenching abilities make them useful for the detection of particular biomarkers [20]. In addition, Gold nanoparticles display high X-ray absorption coefficient which can be used in radiotherapy sensitizers and as imaging agents for tomography [21,22]. Therefore, taking advantage of these unique properties in combination with an effective surface binding method, gold nanoparticles act as an effective nanotheranostics in personal medicine [23].

b) Polymeric Nanoparticles: Clinically the most commonly used FDA approved nanoparticles are polymer-based and are readily available in the market [24]. Such polymeric nanopar- ticles include PEGylated, albumin-encapsulated, liposomal and lipid encapsulated drugs $[25,26]$ as given in Table 1 . Polymeric nanoparticles are composed of amphiphilic substances and are used as imaging agents or in chemotherapeutics and provides an external surface [27-30] for binding of ligands for targeting disease specific biomarkers [31]. For successful treatment, polymeric nanoparticles must remain in the blood stream long enough to generate an immune response [32]. An example of such nanomaterial is PEGylated conjugate which is composed of hyaluronic acid (P-HA-NPs) and is capable of carrying hydrophobic substances into the intracellular regions of tumor cells [33]. P-HA-NPs display low toxicity and accumulates inside tumor cells and then targets an antigen that is overexpressed on the effected cells $[34,35]$. For disease diagnosis, P-HA-NPs surfaces were coated with a specific dye for tumor visualization [36].

Table 1: US FDA-approved Polymeric nanoparticles.

\begin{tabular}{|c|c|c|c|c|}
\hline Commercial Name & Composition & Drug & Indication & Company \\
\hline DaunoXome & Lipid & Daunorubicin & $\begin{array}{c}\text { HIV-associated Kaposi's } \\
\text { sarcoma }\end{array}$ & Celgene \\
\hline Abraxane & $\begin{array}{c}\text { Nanoparticulate } \\
\text { albumin }\end{array}$ & Paclitaxel & Metastatic breast cancer & Corporation [28] \\
\hline Oncaspar & PEGylated & Asparaginase & Acute lymphoblastic leukemia & Enzon pharmaceuticals [29] \\
\hline Doxil & PEGylated liposome & Doxorubicin hydrochloride & Ovarian cancer & Janssen \\
biotech, inc [30]
\end{tabular}

c) Magnetic Nanoparticles: Since magnetic nanoparticles can easily be imaged by MRI so they are commonly used for diagnosis and for effective drug delivery [37]. Though MRI is an effective method for visualizing tissues deep inside the body with high resolution, but it needs external agents that can enhance the contrast to depict things clearly [38]. There are numerous metallic nanoparticles which can be used to increase the contrast by increasing the photon relaxation time [39] Some metal ions that possess this ability are paramagnetic gadolinium, manganese and super-paramagnetic iron oxide [40]. A lot of gadolinium-based contrast agents for MRI are already FDA approved [41]. Iron oxide nanoparticles are mostly employed because of their good magnetic properties, large surface area to volume ratio and their ability to deliver drugs which makes it suitable for nanotheranostics [42]. Micelles and liposomes on the other hand were reported to be more effective imaging agents and also displayed improved drug delivery therefore were more popular in nanotheranostics applications [43].

\section{Mechanisms in Nanotheranostics}

There are several mechanisms through which different nanoparticles or nanoconjugates detect and destroys the cancerous cells some of which are as follows:

a) Smart Nanotheranostics: Smart nanotheranostics is the mechanism in which the specific nanoparticles with unique structural conformation get activated in response to certain biological conditions or internal stimuli in order to identify or deliver the drug to the designated target [44].

b) PH Responsive Nanotheranostics: In response to changes in PH around the areas where the tumors are localized versus the normal tissues, nanoparticles after entering the effected cells become entrapped into lysosomes which are sensitive to PH changes [45]. The procedure relies on the attachment of PH sensitive molecules called linkers. In order to stabilize nanoparticles [46]. Examples of such linkers includes acotinyl, gold nanoparticles conjugated with polyamidoamine dendrimers which displayed good contrast in computed tomography [47]. In addition, dendrimers could be modified with folic acid for high tumor targeting [48]. Another example is of doxorubicin attached to particles with a PH sensitive molecule Cis-aconitic anhydride and the release of drugs were triggered or controlled by acidic environment but not an in vivo case has yet been reported [49]. Organic nanoparticles like bovine serum albumin were linked by cis-aconityl PH sensitive linkers to 
porphyrin photosensitizer pheophorbide and the nanoparticles were made stable by graphene oxide attachment [50]. Though this has great screening and tumor targeting properties, but still encapsulation is required since prophrins are hydrophobic but possess fluorescent and therapeutic efficiency [51]. Several other nanoconjugates like MCDION-Se displays good contrast in MRI and provides limotherapy and chemodynamic at the same time [52]. This specific nanoconjugate is composed of manganese carbonate-deposited iron oxide core with a selenium surface attached to the core through polyethylenimin which imparts an overall negative charge [53]. Another reported example is of biodegradable magnetic mesoporous nanocubic nanoconjugates which when exposed to external magnetic field can generate hyperthermia and had the ability to kill cancerous cells by the production of $\mathrm{H} 2 \mathrm{O} 2$ and ascorbate radical when incorporated with vitamin C [54].

c) ROS Responsive Nanotheranostics: In this mechanism's generation of reactive oxygen species by the cancerous cells act as a biological trigger in nanotheranostics however this mechanism is not possible in oxygen deficient zones [55]. For this approach nanoparticles are designed to allow the release of drugs in response to generation of ROS for an effective chemotherapeutic treatment and also ensures no harm to the nearby normal cells [56]. However, there are different conditions where theranostics based on ROS were implemented. For example, a nanotheranostics system based on boronated maltodextrin works by releasing 4- hydroxybenzyl alcohol in the presence of H2O2 and generate carbon dioxide bubbles for ultrasound imaging due to its echogenic properties [57].

d) Enzyme Responsive Nanotheranostics: this theranostics mechanism depends on enzyme responsiveness [58]. An example of which is ferratin based nanocages that are responsive towards $\mathrm{pH}$ and matrix metalloproteinase MMP-13 activity in osteoarthritis effected individuals [59]. The process is based upon the ability of ferratin to attack collagen II after being genetically modified and a peptide was added in it since the microenvironment of joint on osteoarthritis is acidic and MMP-13 is overexpressed [60]. To make these particles work for enzyme-based diagnostics, MMP-13 peptide which were cleavable plus near-infrared Nir dye cy5.5 and quencher were conjugated [61]. This modification made the process of diagnosis much easier and faster as MMP-13 causes the separation of quencher and dye resulting in the emission of detection signal [62]. Joints have protein matrix that are quite dense and for the nanoparticles to diffuse, their sizes was kept 20nm [63]. When administered through intra articular injection these particles showed good detection of high expression of MMP-13 and therapeutic abilities [64]. Another example is the overexpression of CD44 receptors in cancer cells which were targeted by a carrier HACe6 DoX that contained hyaluronic acid ultrastructure [65]. For enzyme sensitive detection these particles were modified with Ce6 and DOX via hydrazine bond and tumor environment is marked with the overexpression of hyaluronidase which made the release of these chemotherapeutics and photosensitizers faster [66]. Another method that can be used to identify and target cancer cells is the detection of glutathione which is overly expressed in cancer cells and can be used for development of nanotheranostics [67]. Besides, another reported method is the generation of hydrophobic drugs conjugated with hydrophilic polymers [68]. The monomers that are produced are amphiphilic and cause nanoparticles to assemble themselves. This principal is used to create therapeutics and multimodal imaging nanotheranostics [69].

\section{Conclusion}

Thus, nanotheranostics offers promising future in revolutionizing the field of nanomedicine by designing novel nanobased conjugates for effective diagnosis and targeting of cancerous cells by employing unique responsive mechanisms such as enzymebased, PH-based, ROS-based etc., for therapeutics and multimodal imaging nanotheranostics.

\section{References}

1. Kelkar SS, Reineke TM (2011) Theranostics: combining imaging and therapy. Bioconjugate chemistry 22(10): 1879-1903.

2. Svenson S (2013) Theranostics: are we there yet? Molecular pharmaceutics 10(3): 848-856.

3. Jeelani S, Reddy RJ, Maheswaran T, Asokan GS, Dany A, et al. (2014) Theranostics: A treasured tailor for tomorrow. Journal of pharmacy \& bioallied sciences 6(1): 6-8.

4. Picard FJ, Bergeron MG (2002) Rapid molecular theranostics in infectious diseases. Drug discovery today 7(21): 1092-1101.

5. Chen X, Wong S (Eds) (2014) Cancer theranostics. Academic Press.

6. Lee DY, Li KC (2011) Molecular theranostics: a primer for the imaging professional. American Journal of Roentgenology 197(2): 318-324.

7. Mura S, Couvreur P (2012) Nanotheranostics for personalized medicine. Advanced drug delivery reviews, 64(13): 1394-1416.

8. Muthu MS, Leong DT, Mei L, Feng SS (2014) Nanotheranosticsapplication and further development of nanomedicine strategies for advanced theranostics. Theranostics 4(6): 660 .

9. Sonali MKV, Singh RP, Agrawal P, Mehata AK, Datta Maroti Pawde N, et al. (2018) Nanotheranostics: emerging strategies for early diagnosis and therapy of brain cancer. Nanotheranostics 2(1): 70

10. Lammers T, Kiessling F, Hennink WE, Storm G (2010) Nanotheranostics and image-guided drug delivery: current concepts and future directions. Molecular pharmaceutics 7(6): 1899-1912.

11. Wang LS, Chuang MC, Ho JAA (2012) Nanotheranostics-a review of recent publications. International journal of nanomedicine 7: 46794695.

12. Lim EK, Kim T, Paik S, Haam S, Huh YM, et al. (2015) Nanomaterials for theranostics: recent advances and future challenges. Chemical reviews 115(1): 327-394.

13. Huang H, Lovell JF (2017) Advanced functional nanomaterials for theranostics. Advanced functional materials 27(2): 1603524 
14. Peng H, Liu X, Wang G, Li M, Bratlie KM, et al. (2015) Polymeric multifunctional nanomaterials for theranostics. Journal of Materials Chemistry B 3(34): 6856-6870.

15. Jin G, Cheah P, Qu J, Liu L, Zhao Y (2020) Applications of Nanomaterials for Theranostics of Melanoma. Journal of Nanotheranostics, 1(1), 39-55.

16. Cabral RM, Baptista PV (2014) Anti-cancer precision theranostics: a focus on multifunctional gold nanoparticles. Expert review of molecular diagnostics 14(8): 1041-1052.

17. Gao Q Zhang J, Gao J, Zhang Z, Zhu H, et al. (2021) Gold nanoparticles in cancer theranostics. Frontiers in Bioengineering and Biotechnology 6(40): 5134-5151.

18. D’Acunto M, Cioni P, Gabellieri E, Presciuttini G (2021) Exploiting gold nanoparticles for diagnosis and cancer treatments. Nanotechnology 32(19): 192001.

19. Huang X, Jain PK, El-Sayed IH, El-Sayed MA (2008) Plasmonic photothermal therapy (PPTT) using gold nanoparticles. Lasers in medical science 23(3): 217-228.

20. Kim HS, Lee DY (2017) Photothermal therapy with gold nanoparticles as an anticancer medication. Journal of Pharmaceutical Investigation 47(1): 19-26

21. Rancoule C, Magné N, Vallard A, Guy JB, Rodriguez Lafrasse C, et al. (2016) Nanoparticles in radiation oncology: From bench-side to bedside. Cancer letters 375(2): 256-262.

22. Liang G, Jin X, Zhang S, Xing D (2017) RGD peptide-modified fluorescent gold nanoclusters as highly efficient tumor-targeted radiotherapy sensitizers. Biomaterials 144: 95-104.

23. Song G, Cheng L, Chao Y, Yang K, Liu Z (2017) Emerging nanotechnology and advanced materials for cancer radiation therapy. Advanced Materials 29(32): 1700996

24. Indoria S, Singh V, Hsieh MF (2020) Recent advances in theranostic polymeric nanoparticles for cancer treatment: A review. International Journal of pharmaceutics 582: 119314.

25. Kenry Yeo T, Manghnani PN, Middha E, Pan Y, Chen H, et al. (2020) Mechanistic understanding of the biological responses to polymeric nanoparticles. ACS nano 14(4): 4509-4522.

26. Wang Z, Niu G, Chen X (2014) Polymeric materials for theranostic applications. Pharmaceutical research 31(6): 1358-1376.

27. Cole JT, Holland NB (2015) Multifunctional nanoparticles for use in theranostic applications. Drug delivery and translational research 5(3): 295-309.

28. Gou Y, Miao D, Zhou M, Wang L, Zhou H, et al. (2018) Bio-inspired protein-based nanoformulations for cancer theranostics. Frontiers in pharmacology 9: 421.

29. Goswami U, Dutta A, Raza A, Kandimalla R, Kalita S, et al. (2018) Transferrin-copper nanocluster-doxorubicin nanoparticles as targeted theranostic cancer Nanodrug. ACS applied materials \& interfaces 10(4): 3282-3294

30. Zhang F, Zhu L, Liu G, Hida N, Lu G, et al. (2011) Multimodality imaging of tumor response to doxil. Theranostics, 1: 302-309.

31. Chen F, Ehlerding EB, Cai W (2014) Theranostic nanoparticles. Journal of nuclear medicine 55(12): 1919-1922.

32. Choi KY, Liu G, Lee S, Chen X (2012) Theranostic nanoplatforms for simultaneous cancer imaging and therapy: current approaches and future perspectives. Nanoscale 4(2): 330-342.

33. Ryu JH, Lee S, Son S, Kim SH, Leary JF, et al. (2014) Theranostic nanoparticles for future personalized medicine. Journal of controlled release 190: 477-484.

34. Jo SD, Ku SH, Won YY, Kim SH, Kwon IC (2016) Targeted nanotheranostics for future personalized medicine: recent progress in cancer therapy. Theranostics 6(9): 1362.
35. Kim TH, Lee S, Chen X (2013) Nanotheranostics for personalized medicine. Expert review of molecular diagnostics 13(3): 257-269.

36. Sharma R, Mody N, Vyas SP (2017) Bioinspired nanotheranostics for cancer management. In Biopolymer-Based Composites Woodhead Publishing pp. 269-288.

37. Yoo D, Lee JH, Shin TH, Cheon J (2011) Theranostic magnetic nanoparticles. Accounts of chemical research 44(10): 863-874.

38. Gobbo OL, Sjaastad K, Radomski MW, Volkov Y, Prina Mello A (2015) Magnetic nanoparticles in cancer theranostics. Theranostics 5(11): 1249.

39. Angelakeris M (2017) Magnetic nanoparticles: A multifunctional vehicle for modern theranostics. Biochimica et Biophysica Acta (BBA)-General Subjects 1861(6): 1642-1651.

40. Singh A, Sahoo SK (2014) Magnetic nanoparticles: a novel platform for cancer theranostics. Drug discovery today 19(4): 474-481.

41. Zhou Z, Lu ZR (2013) Gadolinium-based contrast agents for magnetic resonance cancer imaging. Wiley Interdisciplinary Reviews: Nanomedicine and Nanobiotechnology 5(1): 1-18

42. Huang J, Zhong X, Wang L, Yang L, Mao H (2012) Improving the magnetic resonance imaging contrast and detection methods with engineered magnetic nanoparticles. Theranostics 2(1): 86.

43. Ahmed SE, Martins AM, Husseini GA (2015) The use of ultrasound to release chemotherapeutic drugs from micelles and liposomes. Journal of drug targeting 23(1): 16-42.

44. Feng L, Dong Z, Tao D, Zhang Y, Liu Z (2018) The acidic tumor microenvironment: a target for smart cancer nano-theranostics. National Science Review, 5(2): 269-286.

45. Kumar EP, Um W, Park JH (2020) Recent Developments in Pathological $\mathrm{P}^{\mathrm{H}}$ Responsive Polymeric Nanobiosensors for Cancer Theranostics. Frontiers in Bioengineering and Biotechnology 8: 601586.

46. Parodi A, Rudzinska M, Leporatti S, Anissimov Y, Zamyatnin AA (2020) Smart nanotheranostics responsive to pathological stimuli. Frontiers in Bioengineering and Biotechnology 8: 503.

47.Singh A, Amiji MM (Eds.) (2018) Stimuli-responsive drug delivery systems. Royal Society of Chemistry.

48. Saluja V, Mankoo A, Saraogi GK, Tambuwala MM, Mishra V (2019) Smart dendrimers: Synergizing the targeting of anticancer bioactives. Journal of Drug Delivery Science and Technology 52: 15-26.

49. Ma Y, Fan X, Li L (2016) $\mathrm{P}^{\mathrm{H}}$ Sensitive polymeric micelles formed by doxorubicin conjugated prodrugs for co-delivery of doxorubicin and paclitaxel. Carbohydrate polymers 137: 19-29.

50. Battogtokh G, Ko YT (2015) Active-targeted $\mathrm{P}^{\mathrm{H}}$-responsive albumin photosensitizer conjugate nanoparticles as theranostic agents. Journal of Materials Chemistry B 3(48): 9349-9359.

51. Almeida Marrero V, van de Winckel E, Anaya Plaza E, Torres T, de la Escosura A (2018) Porphyrinoid biohybrid materials as an emerging toolbox for biomedical light management. Chemical Society Reviews 47(19): 7369-7400.

52. Xiao J, Zhang G, Xu R, Chen $H$, Wang $H$, et al. (2019) A $\mathrm{P}^{\mathrm{H}}$ responsive platform combining chemodynamic therapy with limotherapy for simultaneous bioimaging and synergistic cancer therapy. Biomaterials 216: 119254

53. Xiao J, Zhang G, Xu R, Chen $\mathrm{H}$, Wang $\mathrm{H}$, et al. (2019) A $\mathrm{P}^{\mathrm{H}}$ responsive platform combining chemodynamic therapy with limotherapy for simultaneous bioimaging and synergistic cancer therapy. Biomaterials 216: 119254

54. Li H, Xia P, Pan S, Qi Z, Fu C, et al. (2020) The advances of ceria nanoparticles for biomedical applications in orthopaedics. International Journal of Nanomedicine 15: 7199. 
55. Malla R, Kamal MA (2021) ROS-Responsive Nanomedicine: Towards Targeting the Breast Tumor Microenvironment. Current Medicina Chemistry.

56. Zhou Z, Ni K, Deng H, Chen X (2020) Dancing with reactive oxygen species generation and elimination in nanotheranostics for disease treatment. Advanced Drug Delivery Reviews 158: 73-90.

57. Chen M, Liu D, Liu F, Wu Y, Peng X, et al. (2021) Recent advances of redoxresponsive nanoplatforms for tumor theranostics. Journal of Controlled Release.

58. Yameen B, Wu J, Vilos C, Whyte A, Werstler D, et al. (2016) Stimuliresponsive nanotheranostics. In Nanotheranostics for Personalized Medicine pp. 267-296.

59. Zhang B, Tang G, He J, Yan X, Fan K (2021) Ferritin nanocage: a promising and designable multi-module platform for constructing dynamic nanoassembly-based drug nanocarrier. Advanced Drug Delivery Reviews: 113892

60. Mehta S, He T, Bajpayee AG (2021) Recent advances in targeted drug delivery for treatment of osteoarthritis. Current opinion in rheumatology 33(1): 94-109.

61. Wang Z, Wang S, Wang K, Wu X, Tu C, et al. (2021) Stimuli-Sensitive Nanotherapies for the Treatment of Osteoarthritis. Macromolecular Bioscience: 2100280.

62. Parodi A, Rudzinska M, Leporatti S, Anissimov Y, Zamyatnin AA (2020) Smart nanotheranostics responsive to pathological stimuli. Frontiers in Bioengineering and Biotechnology 8: 503.
63. Mata R, Yao Y, Cao W, Ding J, Zhou T, et al. (2021) The Dynamic Inflammatory Tissue Microenvironment: Signality and Disease Therapy by Biomaterials. Research (Washington, DC) 4189516.

64. Lin X, Xie J, Niu G, Zhang F, Gao H, et al. (2011) Chimeric ferritin nanocages for multiple function loading and multimodal imaging. Nano letters 11(2): 814-819.

65. Parodi A, Rudzinska M, Leporatti S, Anissimov Y, Zamyatnin AA (2020) Smart nanotheranostics responsive to pathological stimuli. Frontiers in Bioengineering and Biotechnology 8: 503.

66. Zhao Q, Liu J, Zhu W, Sun C, Di D, et al. (2015) Dual-stimuli responsive hyaluronic acid-conjugated mesoporous silica for targeted delivery to CD44-overexpressing cancer cells. Acta biomaterialia 23: 147-156.

67. Wang Y, Zu M, Ma X, Jia D, Lu Y, et al. (2020) Glutathione-responsive multifunctional "Trojan Horse" nanogel as a nanotheranostic for combined chemotherapy and photodynamic anticancer therapy. ACS Applied Materials \& Interfaces 12(45): 50896-50908.

68. Manandhar S, Sjöholm E, Bobacka J, Rosenholm JM, Bansal KK (2021) Polymer-Drug Conjugates as Nanotheranostic Agents. Journal of Nanotheranostics 2(1): 63-81.

69. Cai H, Dai X, Wang X, Tan P, Gu L, et al. (2020) A nanostrategy for efficient imaging-guided antitumor therapy through a stimuli-responsive branched polymeric prodrug. Advanced Science 7(6): 1903243. 